\title{
The effect of niobium doping on lithium manganese oxide surfaces, $\mathrm{LiMn}_{2-x} \mathrm{Nb}_{\mathrm{x}} \mathrm{O}_{4}$ : DFT study
}

\author{
B Ramogayana, KP Maenetja, PE Ngoepe
}

Materials Modelling Centre, School of Physical and Mineral Sciences, University of Limpopo, Private Bag x1 106, Sovenga 0727, South Africa Email: brian.ramogayana@ul.ac.za

\begin{abstract}
Lithium manganese oxide $\left(\mathrm{LiMn}_{2} \mathrm{O}_{4}\right)$ is one of the promising cathode material for lithium-ion batteries (LIBs), however, it suffers from capacity fading mainly due to surface manganese $\left(\mathrm{Mn}^{2+}\right)$ ion dissolution during Charge/discharge processes. Although many studies focused on reducing Mn-dissolution, surface modification has proven to be an ideal method of reducing $\mathrm{Mn}^{2+}$ ion dissolution in secondary Li-ion batteries. In this study, the density functional theory calculations were carried out to study the bulk properties and investigate the effect of $\mathrm{Nb}$ surface doping on major $\mathrm{LiMn}_{2} \mathrm{O}_{4}$ spinel surfaces. Upon surface $\mathrm{Nb}$ doping, we observed a decrease in surface free energy as compared to the surface energies of pure surfaces, indicating that the surface stabilizes upon doping. However, the (001) surface remained the most stable facet, with a similar trend of increasing energies and decreasing stability, i.e. $(001)<(011)$ $<(111)$. Due to the stronger binding energy of $\mathrm{Nb}-\mathrm{O}$ as compared to $\mathrm{Mn}-\mathrm{O}$, doping with $\mathrm{Nb}$ can suppress the Mn dissolution during intercalation and hence improve the electrochemical performance.
\end{abstract}

Keywords: Spinel Surfaces, Density functional theory, Lithium-ion batteries

\section{Introduction}

Lithium manganese oxide $\left(\mathrm{LiMn}_{2} \mathrm{O}_{4}\right)$ emerged as a promising alternative cathode material not only because of its affordability, nontoxicity, safety, and abundance of manganese, but also its high operating potential and high energy density (Bensalah and Dawood, 2016). Most especially, it attracted attention because of its three-dimensional crystal network that allows a smooth reversible diffusion of lithium $\left(\mathrm{Li}^{+}\right)$ions during intercalation (Kim and Marnthiram, 1997; Lee et al., 2007). However, the spinel $\mathrm{LiMn}_{2} \mathrm{O}_{4}$-based Li-ion batteries suffer from capacity fading mainly due to manganese $\left(\mathrm{Mn}^{2+}\right)$ ion dissolution (Hunter, 1981; Park et al., 2011), electrolyte oxidation, and the formation of Jahn-Teller distorted $\mathrm{Li}_{2} \mathrm{Mn}_{2} \mathrm{O}_{4}$ tetragonal phases (Arora et al., 1998; Dai et al., 2013) upon cycling. It's believed that manganese dissolution is the major cause of deterioration since Jahn-Teller distortion and electrolyte oxidation can be controlled by cycling at restricted voltages (Bassett et al., 2019; Xia et al., 1997).

Manganese dissolution is enhanced in the presence of hydrofluoric acid continuously produced by the degradation of commercially used LiPF6-electrolyte (Gnanaraj et al., 2003) at elevated temperatures $\left(\sim 55{ }^{\circ} \mathrm{C}\right)$. The $\mathrm{LiPF}_{6}$ electrolyte salt undergoes oxidative decomposition in the presence of traces of water molecules to form this corrosive hydrofluoric acid (HF). The surface $\mathrm{Mn}^{4+}$ reduces to $\mathrm{Mn}^{3+}$ upon accepting electrons from the decomposition of $\mathrm{LiPF}_{6}$ salt. Hence the $\mathrm{Mn}^{3+}$ ions undergoes disproportionation reaction mechanism outlined by Hunter (Hunter, 1981), through the reaction: $\mathrm{Mn}^{3+}$ (solid) $\rightarrow \mathrm{Mn}^{4+}$ (solid) $+\mathrm{Mn}^{2+}$ (solution)

Cation doping has been recommended to reduce the number of trivalent $\mathrm{Mn}^{3+}$ which can undergo a disproportionation reaction. Various dopants were previously explored, which includes Al (Ding et al., 2011; Xiong et al., 2012), Ti (Lu et al., 2014), Ni
(Amarilla et al., 2007), Nb (Dong et al., 2020), Co (Xu et al., 2020), etc. Among most, $\mathrm{Nb}$ is considered as a potential substitute element for $\mathrm{Mn}$ due to the stronger binding energy of $\mathrm{Nb}-\mathrm{O}$ than $\mathrm{Mn}-\mathrm{O}$; which is beneficial for suppressing surface Mn dissolution during intercalation (Zhao et al., 2011; Dong et al., 2020). Li and coworkers (Li et al., 2012) reported that a small amount of niobium $\left(\mathrm{Nb}^{5+}\right)$ doping on the $\mathrm{Li}_{1.02} \mathrm{Mn}_{2} \mathrm{O}_{4}$ can increase the conductivity and improve the cycling performance of the material. Several other studies on lithium manganese-based cathode materials showed that $\mathrm{Nb}$-doping improves cycling performance, and cycling stability (Hu et al., 2018). Son (Son, 2004) found that $\mathrm{LiNbO}_{3}$ is a good material for the cathode materials for lithium-ion batteries and reported that $\mathrm{LiNbO}_{3}$ has a good function for $\mathrm{Li}^{+}$.

To avoid cation ordering that arise from the strong cation-cation interactions in bulk lattice, which give rise to variations in capacity and cycling stability observed in $\mathrm{LiNi}_{0.5} \mathrm{Mn}_{1.5} \mathrm{O}_{4}$ (Santhanam and Rambabu, 2010; Yang et al., 2013), we explore surface doping with $\mathrm{Nb}$ on the spinel $\mathrm{LiMn}_{2} \mathrm{O}_{4}$ surfaces using density functional theory calculations.

\section{Computational method}

\section{Calculation details}

Spin-polarized density functional theory (DFT) techniques as implemented in the Vienna Ab initio Simulation Package (VASP) (Kresse and Furthmüller, 1996) were used to study the bulk stability and the effect of $\mathrm{Nb}$ surface doping and the interactions with the electrolyte components. All calculations were carried out within the generalized gradient approximation (GGA) using the Perdew, Burke, and Ernzerhof (PBE) exchange-correlation functional (Perdew et al., 1996). The kinetic energy cut-off was fixed at $560 \mathrm{eV}$ for the expansion of the Kohn-Sham (KS) valence 
states. A $\Gamma$-centred Monkhorst-Pack grid of 555 and $551 k$-points was used for the integration in the reciprocal space of all surfaces. Phonon code (Parlinski et al., 1997) as implemented in Materials design within MedeA software was used to evaluate the vibrational properties of the material.

The core electrons and their interaction with the valence electrons were described using the projector augmented-wave (PAW) method (Blöchl, 1994) in the implementation of Kresse and Joubert (Kresse and Joubert, 1999). The semi-empirical method of Grimme with the Becke-Johnson damping [D3-(BJ)] (Grimme et al., 2011; Grimme et al., 2010) was also included in our calculations to model the long-range dispersion interactions, which are required to describe the surfaces properly (Santos-Carballal et al., 2014; D. Santos-Carballal et al., 2016).

Gaussian smearing with a width of $0.05 \mathrm{eV}$ was set to improve the convergence of the Brillioun zone integrations during geometry optimizations (Grimme et al., 2010). The tetrahedron method with Blchl corrections was used to obtain accurate electronic and magnetic properties as well as total energies (Blöchl et al., 1994). The Hubbard correction (Anisimov et al., 1992) in the formulation of Dudarev and co-workers (Dudarev et al., 1998) was applied to improve the description of the localized $3 d$ Mn electrons. We have used the effective parameter $\mathrm{U}_{\text {eff }}=4.0 \mathrm{eV}$, which is within the range of values reported in the literature (Bhattacharya and Wolverton, 2013; Karim et al., 2013; Ouyang et al., 2010).

\section{Results and discussions}

\section{Structural properties}

The calculated equilibrium lattice parameter and heats of formation of spinel $\mathrm{LiMn}_{2} \mathrm{O}_{4}$ structure are shown in Table I. It was found that the equilibrium lattice parameter is in good agreement with both previously reported theoretical and experimental results (Chitra et al., 1998; Strobel et al., 2004). The spinel $\mathrm{LiMn}_{2} \mathrm{O}_{4}$ as shown in Figure 1, is a face-centered cubic crystal structure with a space group (No. 227) (Hill et al., 1979). The structure consists of a cubic close-packed array of oxygen atoms occupying the $32 e$ sites, where lithium and manganese atoms occupy one-eighth of the tetrahedral (8a) sites and one-half of the octahedral (16d) sites, respectively (Mishra and Ceder, 1999).

The heat of formation used to predict the stability of the material was calculated using the equation:

$$
\Delta H_{f}=E_{\mathrm{LiMn}_{2} \mathrm{O}_{4}}-\sum_{i} x_{i} E_{i}
$$

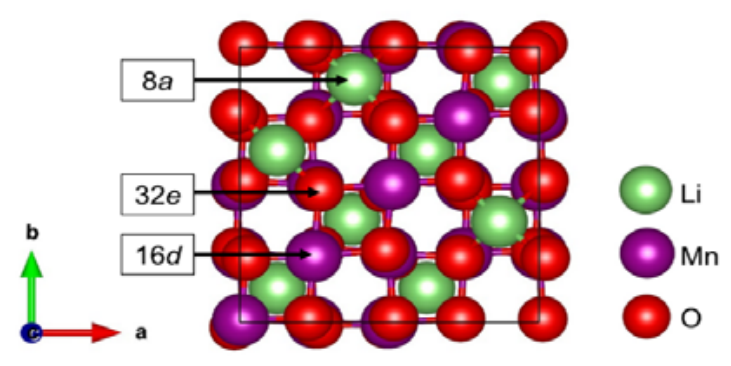

Figure 1: Schematic diagram of the conventional unit cell of $\mathrm{LiMn}_{2} \mathrm{O}_{4}$, showing the $8 \mathrm{a}$ tetrahedral, 16d octahedral, and 32e sites

where, and (which represent, and ) are the total energies of pristine $\mathrm{LiMn}_{2} \mathrm{O}_{4}$ bulk and elemental $\mathrm{Li}, \mathrm{Mn}$, and $\mathrm{O}$ in their respective ground states, while refer to the fractional concentration of each constituent element. The lowest negative value of heats of formation indicates the most stable, while the highest indicates instability of the material. The calculated heats of formation are negative which compares well with reported literature (see Table 1).

\section{Elasticity}

To study the elasticity of the $\mathrm{LiMn}_{2} \mathrm{O}_{4}$ spinel bulk, the elastic constants, moduli, anisotropy, and Pugh's ratio were calculated at the strain of 0.005 (see Table 2). Mechanical stability criteria of a cubic system as outlined elsewhere (Mahlangu, 2013) is given as follows:

$$
C_{44}>0, C_{11}>C_{44} \text {, and } C_{11}+2 C_{12}>0, \mathrm{C}^{\prime}=1 / 2\left(\mathrm{C}_{11}-\mathrm{C}_{12}\right)
$$

where $\mathrm{C}_{11}, \mathrm{C}_{12}$, and $\mathrm{C}_{44}$ are the independent elastic constants. The calculated elastic constants are all positive and obey the mechanical stability criteria for cubic crystals outline in equation 3.2, for example, $C_{11}+2 C_{12}=263.98>0$. Also, the calculated tetragonal shear moduli (C') was found to be $77.06 \mathrm{GPa}$, which is positive and this indicates the stability of the material.

Furthermore, we analyzed Pugh's ratio, which is a ratio of the bulk to shear moduli which indicates the brittleness or the ductility of the material (Wu et al., 2007). A higher value of $\mathrm{B} / \mathrm{G}$ ratio indicates the ductility whereas a low value less than 1.75 indicates the brittleness of the material (If the ratio $\mathrm{B} / \mathrm{G}>1.75$, then the material is ductile and otherwise brittle). The calculated $\mathrm{B} / \mathrm{G}$ ratio was found to be 1.03 , which is less than 1.75 , thus the structure is brittle. Then, we calculated the anisotropy factor (A), which measures the degree of anisotropy in a solid. Anisotropic solid has a value of 1 and measures the degree of elastic anisotropy when the value is less or

Table 1: Calculated lattice parameters and heats of formation for spinel $\mathrm{LiMn}_{2} \mathrm{O}_{4}$ bulk structure

\begin{tabular}{lcccc}
\hline & This Study & \multicolumn{2}{c}{ References } \\
\cline { 3 - 5 } & & Theoretical & Experimental \\
\hline Lattice parameters & 8.247 & (Santos-Carballal et al., 2018) & (karim et al., 2013) & (Ishizawa et al., 2014) \\
Heat of formation & & 8.350 & 8.43 & 8.25 \\
& -1398.56 & (Yamaguchi et al., 1999) & (Wang et al., 2005) \\
\hline
\end{tabular}


Table 2: Elastic constants $\left(\mathrm{C}_{\mathrm{ij}}\right)$ bulk moduli $(\mathrm{B})$, tetragonal shear moduli (C'), shear moduli (G), Young's moduli (Y), anisotropic ratio (A), and Pugh's ratio (K)

\begin{tabular}{llccccc}
\hline Material & \multicolumn{2}{l}{ Elastic constants (GPa) } & \multicolumn{3}{c}{ Moduli } \\
\hline $\mathrm{LiMn}_{2} \mathrm{O}_{4}-$ & $C_{11}$ & 190.75 & $B$ & 88.00 & $C$ & 77.06 \\
normal spinel & $C_{12}$ & 36.63 & $G$ & 85.09 & $A$ & 2.34 \\
& $C_{44}$ & 90.45 & $Y$ & 193.06 & $B / G$ & 1.03 \\
\hline
\end{tabular}

greater than unity ( Pugh, 1954). The value is calculated using the following equation:

$$
A=\frac{2 C_{44}}{C_{11}-C_{12}}
$$

The calculated anisotropy (A) shown in Table 2, is greater than 1, which indicates that the bulk structures are elastically anisotropic materials.

\section{Phonon dispersion and density of states}

To study the vibrational stability, phonon dispersion calculations were performed based on the small displacement method within the harmonic approximation using the PHON code (Parlinski et al., 1997). The Phonon dispersion curves were plotted along the W, $\mathrm{L}, \Gamma, \mathrm{X}$, and $\mathrm{K}$ Brillouin zone directions (see Figure 2a). The curve shows no imaginary vibrations along with all directions, which suggests that the structure is dynamically stable. From the phonon dispersion curve, the acoustic band ranges between 0 and $13 \mathrm{eV}$ while the optical band 15 and $18 \mathrm{eV}$.

Furthermore, we explored the electronic stability and electronic arrangement. The density of states (DOS) was calculated, and the states were summarized in Figure $2 b$.

It can be noted that there are no states observed at the Fermi $\left(E_{\mathrm{f}}\right)$, which indicates that the structure shows no metallic behavior, hence a semiconductor. Instead, a pseudo-gap was observed at the $E_{\mathrm{f}}$ which indicates that the structure is electronically stable. "Pseudo" means "false, unreal or fake", this pseudogap can indicate the stability of the material in density of states (DOS). Figure $3 \mathrm{~b}$ also describes the electronic arrangement according to their respective orbitals. The lower peak of the valence band is dominated by the $s$-orbital of the Li atom, ranging around $-18 \mathrm{eV}$. The upper peaks ranging between -7.0 to $-1.5 \mathrm{eV}$ show hybridization between the $\mathrm{Mn} d$-orbitals and the $\mathrm{O} p$-orbital. At the conduction band, the
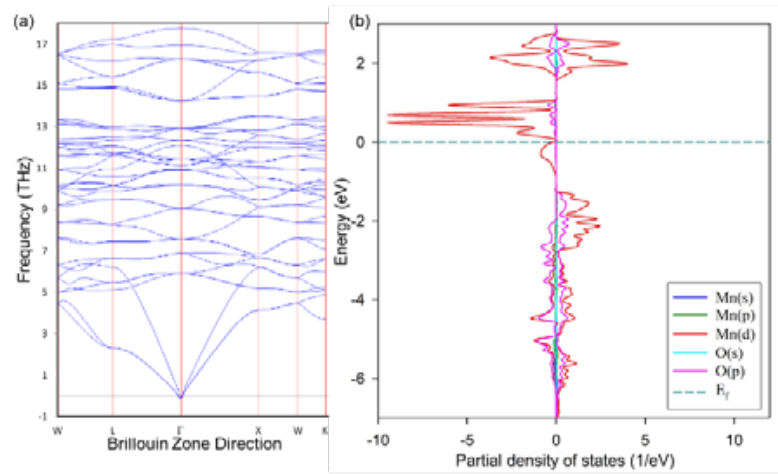

Figure 2: The plot of the (a) phonon dispersion curve and (b) partial density of states (PDOS) for $\mathrm{LiMn}_{2} \mathrm{O}_{4}$ spinel structure

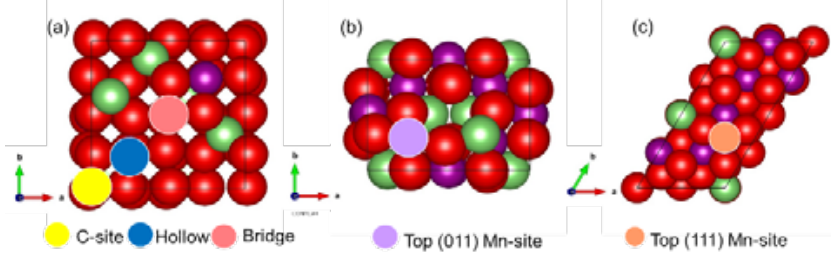

Figure 3: Top view of the most stable surface terminations (a) Li-terminated (001) surface, (b) Li/Mn/O-terminated (011) surface and (c) Li/Mn/O-terminated (111) surface

peaks at around 1.0 and $3.2 \mathrm{eV}$ are dominated by the $d$-orbitals of the Mn atom.

\section{Nb surface doping}

Herein, we study the effect of surface $\mathrm{Nb}$ doping on the major spinel surfaces as compared to the pure pristine facets. With reference to our previous work (Ramogayana et al., 2020), the surface energies of pure pristine facets were calculated for the relaxed and unrelaxed slabs. The calculated energies indicated a trend of increasing surface energies and decreasing stability, i.e. $(001)<(011)<(111)$. In this study, we considered the most stable surface terminations which are the Li-terminated (001) surface, $\mathrm{Li} / \mathrm{Mn} / \mathrm{O}$-terminated (011) surface and $\mathrm{Li} / \mathrm{Mn} / \mathrm{O}$-terminated (111) surface (see Figure 4 below).

Firstly, we explored the most preferred doping sites on the $\mathrm{LiMn}_{2} \mathrm{O}_{4}$ (001), (011), and (111) surfaces. The doping sites investigated include the bridging, hollow, and $\mathrm{C}$-site for the (001) surface while only one site was found for the (011) and (111) surfaces (see Figure 3). The top layer $\mathrm{Mn}$ atom(s) were substituted with $\mathrm{Nb}$ atom(s) and the doped surfaces were then relaxed, allowing all the atoms to move. To study the effect of surface doping, we calculated the surface free energies for the modified surfaces, i.e. for $\mathrm{Nb}$-doped surfaces calculated using:

$$
\sigma=\gamma_{r}+\frac{E_{\mathrm{M}}-E_{\mathrm{r}}-x \cdot E_{\mathrm{Nb}}+x \cdot E_{\mathrm{Mn}}}{A}
$$

where ${ }_{r}$ is the surface energy for the relaxed pristine $\mathrm{LiMn}_{2} \mathrm{O}_{4}$ spinel surface, is the energy of the Nb-doped $\mathrm{LiMn}_{2} \mathrm{O}_{4}$ surface, is the energy of the relaxed pristine. Table 1 summarizes the surface free energies $(\sigma)$ for $\mathrm{Nb}$ doped surfaces with respect to the surface energies of pure pristine surfaces calculated in our previous work. The indicated a trend of increasing surface energies and decreasing stability, i.e. $(001)<(011)<(111)$. The calculated $\sigma$ also show a similar trend, with the (001) surface being the most stable facet upon single $\mathrm{Nb}$ doping. However, the surface free energies are lower as compared to the surface energies for pure spinel surfaces. This decrease in energy as compared to the pure pristine surfaces 
indicates that upon single $\mathrm{Nb}$ doping, the surfaces becomes more stable while the (011) surface remains the most stable facet.

Table 3: Calculated surface free energies $(\sigma)$ for single $\mathrm{Nb}$ doped surfaces as compare to surface energies ( calculated in our previous work

\begin{tabular}{lcc}
\hline & \multicolumn{2}{c}{ Surface free energies $\left(\mathrm{eV} /{ }^{2}\right)$} \\
\hline Surfaces & $\begin{array}{c}\text { Pure } \\
\text { (Ramogayana et al., 2020) }\end{array}$ & Nb doped \\
\hline$(001)$ & 0.037 & 0.017 \\
$(011)$ & 0.050 & 0.018 \\
$(111)$ & 0.052 & 0.063 \\
\hline
\end{tabular}

\section{Density of states}

Furthermore, we compared the total density of states for pure and $\mathrm{Nb}$ doped surfaces (see Figure 4). For pure pristine surfaces, we observed a pseudo gap at the Fermi of (001) surface, which indicates that the facet is the most stable as compared to the other surfaces, and his is in line with the calculated surface energies. However, the (001) surface show a reduced pseudo-gap at the Fermi which indicates that upon doping. From the Inserts, it shows that the $\mathrm{Nb}$ atom doesn't contribute or have any impact on the Fermi level of all the surfaces. The peaks from $\mathrm{Nb}$ atoms in all the surfaces ranges from $1.5-2.5 \mathrm{eV}$, hence we observed the same trend of surface stability before and after $\mathrm{Nb}$ doping.

\section{Conclusion}

The $\mathrm{LiMn}_{2} \mathrm{O}_{4}$ spinel is a face-centered cubic structure with $a=$ 8.175 , which agrees with the reported literature. The calculated elastic constants are all positive and obey the mechanical stability criteria for cubic crystals outline. The calculated $\mathrm{B} / \mathrm{G}$ ratio was found to be 1.03, which is less than 1.75, thus the structure is brittle. The calculated anisotropy (A) is greater than 1, which indicates that the bulk structures are elastically anisotropic. We also explored the density of states (DOS), which showed a pseudo-gap at the Fermi-level, which indicates that the material is electronically stable. We finally plotted the phonon dispersion, which shows no
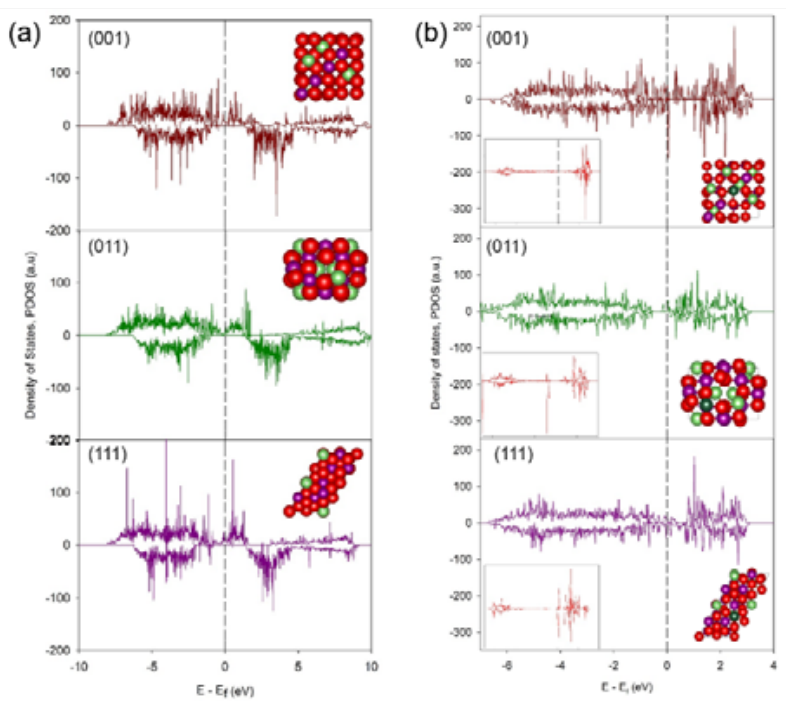

Figure 4: Total density of states for (a) pure pristine $\mathrm{LiMn}_{2} \mathrm{O}_{4}$ and (b) $\mathrm{Nb}$ doped $\mathrm{LiNb}_{0.125} \mathrm{Mn}_{1.875} \mathrm{O}_{4}$ surfaces imaginary vibrations along with all directions, which suggests that the structure is dynamically stable. Upon single $\mathrm{Nb}$ surface doping, the (001) surface remained the most stable facet but the calculated surface free energies as compared to the surface energies of pure pristine surfaces decreased. The partial density of states for $\mathrm{Nb}$ atom doped in the surfaces showed peaks at around $1.5-2.5$ $\mathrm{eV}$, which indicates that the doped $\mathrm{Nb}$ atom has no contribution at the Fermi level, hence the we observed the same trend of surface stability before and after $\mathrm{Nb}$ doping.

\section{Acknowledgments}

This work was carried out at computational facilities of the Materials Modelling Centre (MMC), University of Limpopo, and the Centre for High-Performance Computing in Cape Town. P.E.N also acknowledges the South African Research Chair Initiative of the Department of Science and Technology, the National Research Foundation in Pretoria for funding.

\section{Conflict of interest}

The authors declare that there is no conflict of interest.

\section{ORCII}

Brian Ramogayana

https://orcid.org/0000-0001-6912-1597

Khomotso P. Maenetja

https://orcid.org/0000-0002-3199-0946

Phuti E. Ngoepe

https://orcid.org/0000-0003-0523-5602

\section{References}

1. Bensalah N, Dawood H. 2016. Review on Synthesis, Characterizations, and Electrochemical Properties of Cathode Materials for Lithium Ion Batteries. Journal of Material Science \& Engineering 5, 1 - 21 .

2. Kim J, Marnthiram A. 1997. A manganese oxyiodide cathode for rechargeable lithium batteries. Nature 390, $265-267$.

3. Lee ST, Raveendranath K, Tomy RM, George NA, Jayalekshmi S, Ravi J. 2007. Evidence of band structure modification due to JahnTeller distortion in LixMn2O4 by photoacoustic spectroscopy. Journal of Physics D: Applied Physics 40, 3807 - 3810.

4. Hunter JC. 1981. Preparation of a new crystal form of manganese dioxide: $\lambda-\mathrm{MnO} 2$. Journal of Solid State Chemistry 39, 142 - 147.

5. Park OK, Cho Y, Lee S, Yoo HC, Song HK, Cho J. 2011. Who will drive electric vehicles, olivine or spinel? Energy \& Environmental Science 4, $1621-1633$.

6. Arora P, White RE, Doyle M. 1998. Capacity fade mechanisms and side reactions in lithium-ion batteries. Journal of the Electrochemical Society $145,3647-3667$.

7. Dai Y, Cai L, White RE. 2013. Capacity fade model for spinel LiMn2O4 electrode. Journal of The Electrochemical Society 160, A182 - A190.

8. Bassett KL, Warburton RE, Deshpande S, Fister TT, Ta K, Esbenshade JL, Kinaci A, Chan MK, Wiaderek KM, Chapman KW, Greeley JP. 2019. Operando Observations and First-Principles Calculations of Reduced Lithium Insertion in Au-Coated LiMn2O4. Advanced Materials Interfaces 6, 1801923.

9. Xia Y, Zhou Y, Yoshio M. 1997. Capacity fading on cycling of $4 \mathrm{~V} \mathrm{Li} /$ LiMn2O4 Cells. Journal of The Electrochemical Society 144, 2593 -2600 .

10. Gnanaraj JS, Pol VG, Gedanken A, Aurbach D. 2003. Improving the high-temperature performance of LiMn2O4 spinel electrodes by coating the active mass with $\mathrm{MgO}$ via a sonochemical method. Electrochemistry Communications 11, $940-945$.

11. Ding YL, Xie J, Cao GS, Zhu TJ, Yu HM, Zhao XB. 2011. Enhanced elevated-temperature performance of Al-doped single-crystalline 
LiMn2O4 nanotubes as cathodes for lithium ion batteries. Journal of Physical Chemistry C 115, $9821-9825$.

12. Xiong L, Xu Y, Tao T, Goodenough JB. Synthesis and electrochemical characterization of multi-cations doped spinel LiMn2O4 used for lithium ion batteries. Journal of Power Sources 199, 214 - 219.

13. Lu J, Zhan C, Wu T, Wen J, Lei Y, Kropf AJ, Wu H, Miller DJ, Elam JW, Sun YK, Qiu X. 2014. Effectively Suppressing Dissolution of Manganese from Spinel Lithium Manganate via a Nanoscale SurfaceDoping Approach. Nature communications 5, 1 - 8 .

14. Amarilla JM, Rojas RM, Pico F, Pascual L, Petrov K, Kovacheva D, Lazarraga MG, Lejona I, Rojo JM. 2007. Nanosized LiMYMn2-YO4 $(\mathrm{M}=\mathrm{Cr}$, Co and $\mathrm{Ni})$ spinels synthesized by a sucrose-aided combustion method: Structural characterization and electrochemical properties. Journal of Power Sources 174, 1212 - 1217.

15. Dong S, Zhou Y, Hai C, Zeng J, Sun Y, Shen Y, Li X, Ren X, Sun C, Zhang G, Wu Z. 2020. Understanding electrochemical performance improvement with $\mathrm{Nb}$ doping in lithium-rich manganese-based cathode materials. Journal of Power Sources 462, 228185.

16. Xu D, Yang F, Liu Z, Zeng X, Deng Y, Zheng Y, Lou H, Liao S. 2020. Effects of Co doping sites on the electrochemical performance of LiNi0.5Mn1.5O4 as a cathode material. Ionics 26, 3777-3783.

17. Zhao M, Song X, Wang F, Dai W, Lu X. 2011. Electrochemical performance of single crystalline spinel LiMn2O4 nanowires in an aqueous LiNO3 solution. Electrochimica Acta 56, 5673 - 5678.

18. Li J, Tian Y, Xu C. 2012. Influence of Nb5+ doping on structure and electrochemical properties of spinel Li1.02Mn2O4. Journal of Materials Science \& Technology 28, 817 - 822

19. Hu X, Guo H, Peng W, Wang Z, Li X, Hu Q. 2018. Effects of $\mathrm{Nb}$ doping on the performance of 0.5 $\mathrm{Li} 2 \mathrm{MnO} 3 \cdot 0.5 \mathrm{LiNi} 1 / 3 \mathrm{Co} 1 / 3 \mathrm{Mn} 1 / 3 \mathrm{O} 2$ cathode material for lithium-ion batteries. Journal of Electroanalytical Chemistry 822, 57 - 65 .

20. Son JT. 2004. Novel electrode material for Li ion battery based on polycrystalline LiNbO3. Electrochemistry Communications 10, 990 - 994.

21. Santhanam R, Rambabu B. 2010. Research progress in high voltage spinel LiNi0. 5Mn1.5O4 material. Journal of Power Sources 195, 5442 $-5451$.

22. Yang J, Han X, Zhang X, Cheng F, Chen J. 2013. Spinel LiNi0.5Mn1.5O4 cathode for rechargeable lithium-ion batteries: Nano vs micro, ordered phase ( $P 43$ 32) vs disordered phase (Fd3m). Nano Research 6, 679 - 687.

23. Kresse G, Furthmüller J. 1996. Efficient iterative schemes for ab initio total-energy calculations using a plane-wave basis set. Physical Review B 54, 11169

24. Perdew JP, Burke K, Ernzerhof M. 1996. Generalized gradient approximation made simple. Physical Review Letters 77, 11169 $-11186$.

25. Perdew JP, Burke K, Ernzerhof M. 1996. Generalized gradient approximation made simple. Physical Review letters 77, 11169 $-11186$

26. Parlinski K, Li ZQ, Kawazoe Y. 1997. First-principles determination of the soft mode in cubic ZrO2. Physical Review Letters 78, 4063.

27. Blöchl P. 1994. Projector augmented-wave method. Physical Review B 50, 17953 - 17979.

28. Kresse G, Joubert D. 1999. From ultrasoft pseudopotentials to the projector augmented-wave method. Physical Review B 19, 1758 - 1775 .

29. Grimme S, Ehrlich S, Goerigk L. 2011. Effect of the damping function in dispersion corrected density functional theory. Journal of Computational Chemistry 32, 1456 - 1465.

30. Grimme S, Antony J, Ehrlich S, Krieg H. A consistent and accurate $\mathrm{ab}$ initio parametrization of density functional dispersion correction (DFT-D) for the 94 elements H-Pu. Journal of Chemical Physics 132, 154104.

31. Santos-Carballal D, Roldan A, Grau-Crespo R, de Leeuw NH. 2014. A DFT study of the structures, stabilities and redox behaviour of the major surfaces of magnetite $\mathrm{Fe}_{3} \mathrm{O}_{4}$. Physical Chemistry Chemical Physics 16, $21082-21097$.

32. Santos-Carballal D, Roldan A, de Leeuw NH. 2016. Early Oxidation Processes on the Greigite $\mathrm{Fe}_{3} \mathrm{~S}_{4}$ (001) Surface by Water: A Density Functional Theory Study. Journal of Physical Chemistry C 120, 8616 $-8629$.

33. Santos-Carballal D, Roldan A, Dzade NY, de Leeuw NH. 2018. Reactivity of $\mathrm{CO}_{2}$ on the surfaces of magnetite $\left(\mathrm{Fe}_{3} \mathrm{O}_{4}\right)$, greigite $\left(\mathrm{Fe}_{3} \mathrm{~S}_{4}\right)$ and mackinawite (FeS). Philosophical Transactions. Series A, Mathematical, Physical, and Engineering Sciences 376, 20170065.

34. Blöchl PE, Jepsen O, Andersen OK. 1994. Improved tetrahedron method for Brillouin-zone integrations. Physical Review B 49, 16223 $-16233$

35. Anisimov VI, Korotin MA, Zaanen J, Andersen OK. 1992. Spin bags, polarons, and impurity potentials in $\mathrm{La} 2-\mathrm{xSrxCuO} 4$ from first principles. Physical Review Letters 68, 345 - 348.
36. Dudarev SL, Botton GA, Savrasov SY, Humphreys CJ, Sutton AP. 1998. Electron-energy-loss spectra and the structural stability of nickel oxide: An LSDA+ U study. Physical Review B 57, 1505 - 1509.

37. Bhattacharya J, Wolverton C. 2013. Relative stability of normal vs. inverse spinel for $3 \mathrm{~d}$ transition metal oxides as lithium intercalation cathodes. Physical Chemistry Chemical Physics 15, 6486 - 6498.

38. Karim A, Fosse S, Persson KA. 2013. Surface structure and equilibrium particle shape of the LiMn2O4 spinel from first-principles calculations. Physical Review B 87, 075322-1 - 075322-6.

39. Ouyang CY, Zeng XM, Sljivancanin Z, Baldereschi A. 2010 Oxidation states of $\mathrm{Mn}$ atoms at clean and Al2O3-covered LiMn2O4 (001) surfaces. Journal of Physical Chemistry C 114, 4756 - 4759.

40. Chitra S, Kalyani P, Mohan T, Massot M, Ziolkiewicz S, Gangandharan R, Eddrief M, Julien C. 1998. Physical Properties of LiMn204 Spinel Prepared at Moderate Temperature. Ionics 4, 8 - 15.

41. Strobel P, Rousse G, Ibarra-Palos A, Masquelier C. 2004. Disproportionation of stoichiometric LiMn2O4 on annealing in oxygen. Journal of Solid State Chemistry 177, $1-5$.

42. Hill RJ, Craig JR, Gibbs GV. 1979. Systematics of the spinel structure type. Physics and Chemistry of Minerals 4, 317 - 339.

43. Mishra SK, Ceder G. 1999. Structural stability of lithium manganese oxides. Physical Review B 59, 6120 - 6130.

44. Santos-Carballal D, Ngoepe PE, de Leeuw NH. 2018. Ab initio investigation of the thermodynamics of cation distribution and of the electronic and magnetic structures in the LiMn2O4 spinel. Physical Review B 97, 085126-1 - 085126-11.

45. Ishizawa N, Tateishi K, Oishi S, Kishimoto S. 2014. Bond-length fluctuation in the orthorhombic $3 \times 3 \times 1$ superstructure of LiMn2O4 spinel. American Mineralogist 99, 1528 - 1536.

46. Yamaguchi R, Ikuta H, Wakihara M. 1999. Heat of Formation for LiMyMn2- yO4 (M= Co, Cr, Li, Mg, Ni) Spinel Solid Solution. Journal of Thermal Analysis and Calorimetry 57, 797 - 806.

47. Wang M, Navrotsky A. 2005. Thermochemistry of Li1+xMn2-xO4 $(0 \square \mathrm{x} \square 1 / 3)$ spinel. Journal of Solid State Chemistry 178, 1182 - 1189

48. Mahlangu R, Phasha MJ, Chauke HR, Ngoepe PE. 2013. Structural, elastic and electronic properties of equiatomic PtTi as potential high-temperature shape memory alloy. Intermetallics 33, 27 - 32.

49. Wu ZJ, Zhao EJ, Xiang HP, Hao XF, Liu XJ, Meng J. 2007. Crystal structures and elastic properties of superhard IrN2 and IrNr3 from first principle. Physical Review B 76, 054115.

50. Pugh S. 1954. XCII. Relations between the elastic moduli and the plastic properties of polycrystalline pure metals. The London, Edinburgh, and Dublin Philosophical Magazine and Journal of Science $45,823-843$.

51. Ramogayana B, Santos-Carballal D, Aparicio PA, Quesne MG Maenetja KP, Ngoepe PE, de Leeuw NH. 2020. Ethylene carbonate adsorption on the major surfaces of lithium manganese oxide Li1xMn2O4 spinel $(0.000<\mathrm{x}<0.375)$ : a DFT+U-D3 study. Physical Chemistry Chemical Physics 22, 6763 - 6771.

52. Bao SJ, Liang YY, Zhou WJ, He BL, Li HL. 2006. Synthesis and electrochemical properties of LiAl0. $1 \mathrm{Mn} 1.9 \mathrm{O} 4$ by microwaveassisted sol-gel method. Journal of Power Sources 154, 239 - 245.

53. Amarilla JM, Petrov K, Pico F, Avdeev G, Rojo JM, Rojas RM. 2009. Sucrose-aided combustion synthesis of nanosized LiMn1.99yLiyM0.0104 $(\mathrm{M}=\mathrm{Al} 3+, \mathrm{Ni} 2+, \mathrm{Cr} 3+, \mathrm{Co} 3+, \mathrm{y}=0.01$ and 0.06$)$ spinels: Characterization and electrochemical behavior at 25 and at $55^{\circ} \mathrm{C}$ in rechargeable lithium cells. Journal of Power Sources 191 $591-600$.

54. Zakaria SNA, Hollingsworth N, Islam H, Roffey A, Santos-Carballal D, Roldan A, Bras W, Sankar G, Hogarth G, Holt KB, de Leeuw NH. 2018. Insight into the Nature of Iron Sulfide Surfaces During the Electrochemical Hydrogen Evolution and CO2 Reduction Reactions. ACS Applied Materials \& Interfaces 10, 32078 - 32085.

55. Posada-Pérez S, Santos-Carballal D, Terranova U, Roldan A, Illas F, de Leeuw NH. 2018. $\mathrm{CO}_{2}$ Interaction with Violarite $\left(\mathrm{FeNi}_{2} \mathrm{~S}_{4}\right)$ Surfaces: A Dispersion-Corrected DFT Study. Physical Chemistry Chemical Physics 20, 20439 - 20446.

56. Postica V, Vahl A, Strobel J, Santos-Carballal D, Lupan O, Cadi-Essadek A, de Leeuw NH, Schütt F, Polonskyi O, Strunskus T, Baum M, Kienle L, Adelung R, Faupel F. 2018. Tuning doping and surface functionalization of columnar oxide films for volatile organic compound sensing: experiments and theory. Journal of Materials Chemistry A 6, 23669 - 23682.

57. Ouyang CY, Shi SQ, Lei MS. 2009. Jahn-Teller distortion and electronic structure of LiMn2O4. Journal of Alloys and Compounds 474, 370 - 374 .

58. Tasker PW. 1979. The stability of ionic crystal surfaces . Journal of Physics C: Solid State Physics 12, 4977 - 4984.

59. Watson GW, Kelsey ET, de Leeuw NH, Harris DJ, Parker SC. 1996. Atomistic simulation of dislocations, surfaces and interfaces in $\mathrm{MgO}$. Journal of the Chemical Society, Faraday Transactions 92, 433 - 438. 